\title{
Peritoneal Potassium and pH Measurement in Early Diagnosis of Acute Mesenteric Ischemia in Rats
}

\author{
Mehrdad Hosseinpour ${ }^{1, *}$; Tahere Khamechian ${ }^{1}$; Soraya Shahrokh ${ }^{1}$ \\ ${ }^{1}$ Trauma Research Center, Kashan University of Medical Sciences, Kashan, IR Iran \\ ${ }^{*}$ Corresponding author: Mehrdad Hosseinpour, Trauma Research center, Kashan University of Medical Sciences, Kashan, IR Iran. Tel: +98-3116255368, Fax: +98-3116255368, E-mail: \\ meh_hosseinpour@yahoo.com
}

Received: June 4, 2014; Accepted: July 9, 2014

\begin{abstract}
Background: In contemporary practice, acute mesenteric ischemia (AMI) remains a serious cause of morbidity and mortality in abdominal emergencies.

Objectives: We report the measurement of peritoneal fluid potassium and $\mathrm{pH}$ on a small series of rats that developed extensive AMI following the surgical ligation of superior mesenteric vessels and compare the results with control groups.

Materials and Methods: A total of 32 rats were used in our study. They were divided into four groups with eight rats in each one and received following treatments: group I (G-I), 60-minute controls; group II (G-II), 120-minute controls; group III (G-III), 60-minute cases; and group IV (GIV), 120-minute cases. In case groups, the small bowel mesenteric root was double-ligated and an arrow single-lumen central venous catheter was passed through the skin to the peritoneum. In control groups, the catheter was placed without any intervention. Postoperatively, peritoneal lavage was performed at 60 (G-I, G-III) and 120 minutes (G-II, G-IV).

Results: The mean peritoneal potassium values were $1.3 \pm 0.3,1.97 \pm 1.06,2.14 \pm 0.89$, and $3.28 \pm 0.66 \mathrm{mmol} / \mathrm{L}$ in G-I, G-II, G-III, and G-IV, respectively. There were significant differences between G-III and G-IV $(P=0.002)$, between G-I and G-III $(P=0.024)$, and between G-II and G-IV $(P=0.001)$. The mean value of peritoneal fluid $\mathrm{pH}$ were $7.1 \pm 0.26,6.82 \pm 0.22,6.66 \pm 0.16$, and $6.78 \pm 0.04$ in G-I, G-II, G-III, and G-IV, respectively, which indicated significant differences between G-I and G-III $(\mathrm{P}=0.001)$ and between G-II and G-IV $(\mathrm{P}=0.018)$. There was a significant correlation between peritoneal fluid potassium and intestine ischemic grade $(\mathrm{F}=4.77, \mathrm{P}=0.048)$

Conclusions: Our findings show that for early detection of bowel ischemia, an evaluation of intraperitoneal potassium and $\mathrm{pH}$ was useful and with prolongation of ischemia, potassium changes were more significant.
\end{abstract}

Keywords: Ischemia; Potassium; Acute

\section{Background}

Acute mesenteric ischemia (AMI) remains a serious cause of morbidity and mortality for abdominal emergencies and its incidence continues to raise $(1,2)$. The survival rate has not improved during the past 70 years and the management of extensive disease still poses major problems (3-5). The major reason for these problems is the difficulty in prompt recognizing of the condition before bowel necrosis. Researches have proposed different serum or peritoneal markers (6-9) and various types of imaging $(10,11)$ for the diagnosis of AMI.

\section{Objectives}

We measured the peritoneal fluid potassium and $\mathrm{pH}$ on a small series of rats that developed an extensive AMI following the surgical ligation of superior mesenteric vessels and compared the results with control groups to find the association of these parameters with AMI.

\section{Materials and Methods}

Local research ethics committee approved the study protocol. A total of 32 rats were used in our study to compare the biochemical changes of peritoneal fluid in controls and cases with extensive AMI, which was caused by ligation of mesenteric vessels at the small bowel mesenteric root. The adult albino male Wistar rats (Razi Institute, Tehran, Iran) weighting 200 to 300 g were selected, numbered from 01 to 32, and tattooed on the internal surface of the right ear. The rats were kept in a controlled environment (temperature of $24^{\circ} \mathrm{C}-26^{\circ} \mathrm{C}$ and humidity of $55 \%-65 \%$ ), fed with a commercial pellet diet (Niro-Sahand co, Tabriz, Iran), and allowed access to tap water ad libitum. Feeding was discontinued four hours before surgery. Then they were randomly allocated to four groups of eight animals and treated as follows: group I (G-I), 60-minute controls; group II (G-II), 120-minute controls; group III (G-III), 60-minute cases; and group IV (G-IV), 120-minute cases.

After premedication with $2-\mathrm{mg} / \mathrm{kg}$ intramuscular acepromazine (30 minutes before anesthesia), anesthesia was induced through intramuscular injection of 4-mg/kg xylazine and 40-mg/kg ketamine. The animal

Copyright (C) 2014, Kashan University of Medical Sciences; Published by Kowsar. This is an open-access article distributed under the terms of the Creative Commons Attribution-NonCommercial 4.0 International License (http://creativecommons.org/licenses/by-nc/4.0/) which permits copy and redistribute the material just in noncommercial usages, provided the original work is properly cited. 
was placed in a horizontal dorsal decubitus on the surgical table and its paws were fixed to the extremities of the table with thin ropes. Abdominal hairs were shaved from the abdominis cranialis region and media with an electrical clipper at the site of surgery. Antisepsis of the surgical site was performed with $2 \%$ iodinated alcohol solution. Median laparotomy was performed starting $1 \mathrm{~cm}$ below the xiphoid process, in caudal direction with 3-cm extension. In case groups, after exteriorization of intestinal loops, small bowel mesenteric root was double ligated by silk zero to occlude the mesenteric vessels. Thereafter, an arrow single-lumen central venous catheter (14 GA) was passed through skin and peritoneum and was fixed to skin. Then the abdominal wall was closed and the animals were allowed to recover from anesthesia. In control groups, catheter was placed without any intervention on mesentery. Postoperatively, peritoneal lavage was performed by $200 \mathrm{~mL}$ of warm saline solution at 60 (G-I, GIII) and 120 minutes (G-II, G-IV) and back-washed fluid was collected. Simultaneously, a sample of blood was drawn for measurement of blood potassium. Then animals were sacrificed by cardiac puncture and intestinal tract was removed and fixed in 10\% formalin. Specimens were stained with hematoxylin and eosin and a pathologist, who was unaware of the allocation of the study cases, examined the specimens histopathologically. Blood and peritoneal potassium was measured by flame photometry technique. The $\mathrm{pH}$ of peritoneal fluid was measured by dipstick method. For grading intestinal ischemia, we used Chiu et al. (12) grading scale (Table 1). Quantitative data were reported as mean \pm standard deviation (SD). Data were examined for distribution normality using Kolmogorov-Smirnov test. K-sample Kruskal-Wallis test and ANOVA were used to compare the results. Data were analyzed using SPSS (version 12, SPSS Inc, Chicago, IL, USA) and P value $<0.05$ was considered significant.

\section{Results}

The groups had no deference regarding age. The mean body weight of rats was $235.2 \pm 102.9 \mathrm{~g}$ and there was no significant difference between groups in this regard. Ischemic grade was more severe in G-IV (mean, 3.5 \pm 0.75 ; and mean rank, 12.5) in comparison with G-III (mean, 1.75 \pm 0.46 ; and mean Rank, 4.5) $(\mathrm{P}=0.001)$. Table 2 shows the results of blood and peritoneal fluid potassium measurements in study groups. The mean of peritoneal potassium was $1.3 \pm 0.3,1.97 \pm 1.06,2.14 \pm 0.89$, and $3.28 \pm 0.66$ mmol/L in G-I, G-II, G-III, and G-IV, respectively, which showed a significant differences between G-III and G-IV (P $=0.002), \mathrm{G}-\mathrm{I}$ and G-III $(\mathrm{P}=0.024)$, as well as G-II and G-IV (P $=0.001)$. The mean of blood potassium levels were $9.09 \pm$ 0.55, $9.69 \pm 1.21,9.08 \pm 0.42$, and $10.9 \pm 1.54 \mathrm{mmol} / \mathrm{L}$ in G-I, G-II, G-III, and G-IV, respectively, which showed a significant difference between G-III and G-IV $(\mathrm{P}=0.021)$ as well as G-II and G-IV (P=0.002). There was no significant regression model between peritoneal and blood potassium by ANOVA test.

Table 1. Histopathologic Grading of Intestinal Ischemia

\begin{tabular}{lc}
\hline Description & Grade \\
\hline Normal & 0 \\
\hline Subepithelial Space at the Top of Villus & 1 \\
\hline Epithelial Lifting at the Top of Villus & 2 \\
\hline Massive Epithelial Lifting & 3 \\
\hline Denuded Villus & 4 \\
\hline Disintegration of the Mucosa + Ulceration + Hemorrhage & 5 \\
\hline
\end{tabular}

Table 2. Blood and Peritoneal Fluid Potassium Levels in the Study Groups a, b

\begin{tabular}{|c|c|c|c|c|c|c|c|}
\hline \multicolumn{2}{|c|}{ G-I } & \multicolumn{2}{|c|}{ G-II } & \multicolumn{2}{|c|}{ G-III } & \multicolumn{2}{|c|}{ G-IV } \\
\hline BK & PK & BK & PK & BK & PK & BK & PK \\
\hline 8.64 & 3.58 & 9.79 & 4.1 & 10.53 & 1.02 & 10.25 & 0.62 \\
\hline 9.52 & 2.32 & 10.29 & 3.25 & 9.73 & 1.56 & 8.75 & 0.88 \\
\hline 8.63 & 3.32 & 10.43 & 3.7 & 9.94 & 1.33 & 9.39 & 0.86 \\
\hline 8.64 & 1.77 & 9.56 & 3.31 & 9.85 & 1.19 & 7.97 & 1.04 \\
\hline 9.52 & 2.05 & 13.09 & 4.12 & 10.35 & 1.82 & 8.8 & 2.65 \\
\hline 8.95 & 1.56 & 11.34 & 2.71 & 10.84 & 1.41 & 8.22 & 1.07 \\
\hline 9.59 & 1.11 & 9.44 & 2.74 & 9.11 & 0.82 & 8.6 & 0.72 \\
\hline 9.15 & 1.43 & 13.34 & 2.32 & 9.61 & 1.31 & 8.36 & 1.36 \\
\hline
\end{tabular}

a Abbreviations: G, group; BK, blood potassium; and PK, peritoneal potassium.

$\mathrm{b}_{\text {The measurements are expressed as mmol/L. }}$ 
According the above findings, our results showed that: a) After 120 minutes of abdominal incision in rats without AMI (G-II), no significant changes in peritoneal fluid or blood potassium were seen.

b) After 60 minutes of bowel ischemia (G-III), peritoneal fluid potassium increased; however, there were no significant changes in blood potassium.

c) After 120 minutes of bowel ischemia (G-IV), both blood and peritoneal fluid potassium increased significantly.

Table 3 shows pH measurements in the peritoneal fluid of the study groups. The mean of peritoneal fluid $\mathrm{pH}$ was $7.1 \pm 0.26,6.82 \pm 0.22,6.66 \pm 0.16$, and $6.78 \pm 0.04$ in G-I, G-II, G-III, and G-IV, respectively, which indicated a significant difference between G-I and G-III $(P=0.001)$ as well as $\mathrm{G}-\mathrm{II}$ and $\mathrm{G}-\mathrm{IV}(\mathrm{P}=0.018)$. On the other hand, we had no decrease in $\mathrm{pH}$ after simple laparotomy and prolongation of ischemia had no significant effect on $\mathrm{pH}$ of peritoneal fluid (G-I vs. G-II and G-III vs. G-IV). The ANOVA test showed a significant regression model between peritoneal fluid potassium and intestine ischemic grade $(\mathrm{F}=4.77, \mathrm{P}=$ 0.048) (Figure 1).

\begin{tabular}{lccc}
\hline \multicolumn{4}{l}{ Table 3. Peritoneal Fluid pH } \\
\hline G-I & G-II & G-III & G-IV \\
\hline $\mathbf{6 . 8 2}$ & 6.75 & 6.95 & 6.95 \\
$\mathbf{6 . 5 3}$ & 6.83 & 7.03 & 6.82 \\
$\mathbf{6 . 3 2}$ & 6.81 & 6.86 & 6.9 \\
$\mathbf{6 . 6 4}$ & 6.76 & 6.87 & 6.87 \\
$\mathbf{6 . 7 6}$ & 6.8 & 6.95 & 6.88 \\
$\mathbf{6 . 8 2}$ & 6.79 & 7.17 & 6.99 \\
$\mathbf{6 . 7 3}$ & 6.82 & 7,44 & 6.7 \\
$\mathbf{6 . 7 1}$ & 6.71 & 7.54 & 6.94 \\
$\mathbf{6 . 8 2}$ & 6.75 & 6.95 & 6.95 \\
\hline
\end{tabular}

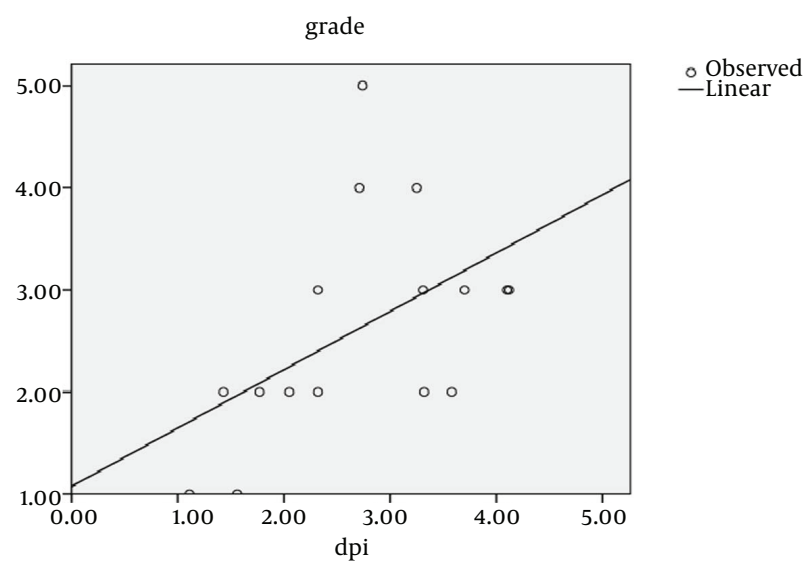

Figure 1. Regression model Between Peritoneal Fluid Potassium and Intestine Ischemic Grade $(\mathrm{F}=4.77, \mathrm{P}=0.048)$

\section{Discussion}

Acute mesenteric ischemia is an abdominal emergency that should be distinguished from other critical conditions. Early diagnosis is the first step in management and its first goal is to find a marker (plasma or peritoneal) with sufficient sensitivity and specificity. Although a high index of suspicion in the setting of a compatible history and physical examination serves as the cornerstone to early diagnosis of AMI, many of the signs and symptoms associated with AMI are common to other intra-abdominal pathologic conditions such as pancreatitis, acute diverticulitis, and small bowel obstruction (13-15).

Extensive experimental researches on intestinal ischemia using various markers were published; however, their clinical relevance has been questioned. The main objective of this study was to examine the results of intraperitoneal lavage for early diagnosis of AMI. The overall results indicated that changes in peritoneal potassium were more pronounced in AMI. The absence of a significant difference in blood potassium in the present study might be explained by self-limiting process of acute ischemia. In general, ischemia is a process of acute inflammation that is self-limiting, i.e. it is able to keep the reaction local and thereby, preventing generalization of the process. On the other hand, kidney's capacity to clear potassium from circulation may be another reason for the absence of hyperkalemia during the early hour of bowel ischemia. Instead, prolonged ischemia can release a large amount of inflammatory mediators into the circulation and commence general deterioration and systemic hypoperfusion, which leads to hyperkalemia. During bowel ischemia, the activity of the plasma membrane energydependent sodium pump is reduced, which results in intracellular accumulation of sodium and efflux of potassium. Therefore, there is a compensatory increase in anaerobic glycolysis in an attempt to maintain the cell's energy source. Consequently, intracellular glycogen stores are rapidly depleted and lactic acid accumulates, which leads to decreased intracellular $\mathrm{pH}$. Diffusion of slight amount of hydrogen ions through cell membrane can lead to decreased extracellular and intraperitoneal fluid $\mathrm{pH}$ after one hour of bowel ischemia. With prolongation of ischemia, there will be a significant increase in intraperitoneal potassium due to increased injury to sodium pumps and increased potassium efflux.

In a similar study, Gonullu et al. (16) showed the same results, but the major difference between our results and their study was significant changes of peritoneal fluid potassium after one hour of ischemia. In Gonullu study, there was no significant difference between sham and 60-minute cases, but our results showed that peritoneal potassium could be used as an ischemic marker after 60 minutes of ischemia. As shown by DeLaurier et al. (17), with prolongation of ischemia to two hours, differences of $\mathrm{pH}$ would not be significant. This can be explained by the protein buffers system mechanism. The most plentiful buffers of the body are the cell's proteins. Thus, this 
intracellular buffer system helps to buffer the extracellular fluid. However, the slow movement of hydrogen and bicarbonate ions through the cell membranes often delays the ability of the intracellular buffers to buffer extracellular acid-base abnormalities for several hours, which would lead to significant differences in $\mathrm{pH}$ between the case and control groups. It seems that if the ischemia is prolonged to more than six hours, irreversible injury and necrosis will ensue. The cell's components are progressively degraded and there would be a widespread leakage of cellular enzymes. Consequently, released intracellular hydrogen ions cause a surge of extracellular hydrogen ions. The major limitation of this study was the manipulation of bowels in comparison to the actual clinical scenario, which might have changed the peritoneal variables.

In summary, our findings showed that for early detection of bowel ischemia, evaluation of intraperitoneal potassium and $\mathrm{pH}$ would be useful and with prolongation of ischemia, changes of potassium would be more significant.

\section{Acknowledgements}

We would like to thank the vice chancellor of Kashan University of Medical Sciences and animal lab personnel for their great contribution in this research.

\section{Authors' Contributions}

Mehrdad Hosseinpour, study concept and design, drafting of the manuscript, critical revision of the manuscript for important intellectual content, and study supervision; Soraya Shahrokh, acquisition of data, analysis and interpretation of data, and statistical analysis; Tahere Khamechian, administrative, technical, and material support.

\section{Funding/Support}

All financial and material support for the research was provided by vice chancellor of Kashan University of Medical Sciences.

\section{References}

1. Oldenburg WA, Lau LL, Rodenberg TJ, Edmonds HJ, Burger CD Acute mesenteric ischemia: a clinical review. Arch Intern Med. 2004;164(10):1054-62.

2. Chang RW, Chang JB, Longo WE. Update in management of mesenteric ischemia. World J Gastroenterol. 2006;12(20):3243-7.

3. Arthurs ZM, Titus J, Bannazadeh M, Eagleton MJ, Srivastava S, Sarac TP, et al. A comparison of endovascular revascularization with traditional therapy for the treatment of acute mesenteric ischemia. J Vasc Surg. 2011;53(3):698-704.

4. Harnik IG, Brandt LJ. Mesenteric venous thrombosis. Vasc Med. 2010;15(5):407-18.

5. Cortese B, Limbruno U. Acute mesenteric ischemia: primary percutaneous therapy. Catheter Cardiovasc Interv. 2010;75(2):283-5.

6. Dundar ZD, Cander B, Gul M, Karabulut KU, Girisgin S. Serum ischemia-modified albumin levels in an experimental acute mesenteric ischemia model. Acad Emerg Med. 2010;17(11):1233-8.

7. Chiu YH, Huang MK, How CK, Hsu TF, Chen JD, Chern CH, et al. D-dimer in patients with suspected acute mesenteric ischemia. Am J Emerg Med. 2009;27(8):975-9.

8. Murray MJ, Gonze MD, Nowak LR, Cobb CF. Serum D(-)-lactate levels as an aid to diagnosing acute intestinal ischemia. Am J Surg. 1994;167(6):575-8.

9. Lammers KM, Innocenti G, Venturi A, Rizzello F, Helwig U, Bianchi GP, et al. The effect of transient intestinal ischemia on inflammatory parameters. Int J Colorectal Dis. 2003;18(1):78-85.

10. Yikilmaz A, Karahan OI, Senol S, Tuna IS, Akyildiz HY. Value of multislice computed tomography in the diagnosis of acute mesenteric ischemia. Eur J Radiol. 2011;80(2):297-302.

11. Menke J. Diagnostic accuracy of multidetector CT in acute mesenteric ischemia: systematic review and meta-analysis. Radiology. 2010;256(1):93-101.

12. Chiu CJ, McArdle AH, Brown R, Scott HJ, Gurd FN. Intestinal mucosal lesion in low-flow states. I. A morphological, hemodynamic, and metabolic reappraisal. Arch Surg. 1970;101(4):478-83.

13. Ellis J, Sarac TP. Acute mesenteric ischemia. Minerva Chir 2010;65(3):297-301.

14. Wyers MC. Acute mesenteric ischemia: diagnostic approach and surgical treatment. Semin Vasc Surg. 2010;23(1):9-20.

15. Bjorck M. Mesenteric vascular disease: problems and progress. Introduction. Semin Vasc Surg. 2010;23(1):1-3.

16. Gonullu D, Yankol Y, Isiman F, Akyildiz Igdem A, Yucel O, Koksoy FN. [pH value and potassium level of diagnostic peritoneal lavage fluid in the early diagnosis of acute mesenteric ischemia secondary to arterial occlusion in rats]. Ulus Travma Acil Cerrahi Derg. 2007;13(4):261-7.

17. DeLaurier GA, Cannon RM, Johnson RH, Jr, Sisley JF, Baisden $\mathrm{CR}$, Mansberger AJ. Increased peritoneal fluid lactic acid values and progressive bowel strangulation in dogs. Am J Surg. 1989;158(1):32-5. 